\title{
THEORY OF INTERSTITIAL OXYGEN IN SILICON AND GERMANIUM
}

\author{
EMILIO ARTACHO AND FELIX YNDURAIN \\ Departamento de Física de la Materia Condensada, C-III \\ Universidad Autónoma de Madrid, 28049 Madrid, Spain.
}

Keywords: Oxygen in covalent semiconductors.

\begin{abstract}
The interstitial oxygen centers in silicon and germanium are reconsidered and compared in an analysis based on the first-principles total-energy determination of the potential-energy surface of the centers, and a calculation of their respective low energy excitations and infrared absorption spectra. The total-energy calculations reveal unambiguously that interstitial oxygen is quantum delocalized, the delocalization being essentially different in silicon and in germanium. Oxygen in silicon lies at the bond center site in a highly anharmonic potential well, whereas in germanium it is found to rotate almost freely around the original Ge-Ge bond it breaks. This different delocalization is the origin of the important differences in the low energy excitation spectra: there is a clear decoupling in rotation and vibration excitations in germanium, giving different energy scales $\left(1 \mathrm{~cm}^{-1}\right.$ for the rotation, $200 \mathrm{~cm}^{-1}$ for the $\nu_{2}$ mode), whereas both motions are non-trivially mixed in silicon, in a common energy scale of around $30 \mathrm{~cm}^{-1}$. The calculation of the vibrational spectra of the defect reveals the existence of vibrational modes (related to the $\nu_{1}$ mode) never been experimentally observed due to their weak infrared activity. It is found that the combination of these modes with the well established $\nu_{3}$ asymmetric stretching ones is the origin of the experimentally well characterized modes at frequencies above the $\nu_{3}$ mode frequency.
\end{abstract}

\section{Introduction}

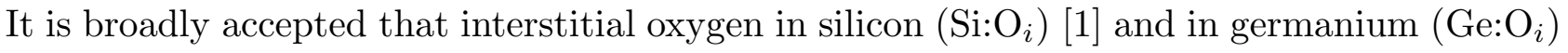
[2-5] represent very similar centers in their geometry and dynamics. The aim of this paper is to show the contrary: the quantitative differences in the low-energy experimental results for

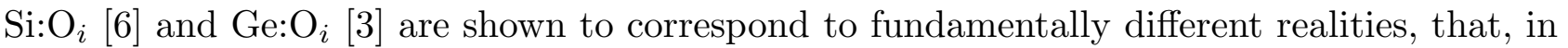
turn, lead us to predict substantial differences in their infrared absorption spectra. The basic physics of both centers as well as their similarities and differences are analyzed and discussed in this work, the essence being graphically depicted in Fig. 1. Oxygen breaks a covalent bond

Fig. 1: Schematic representation of the

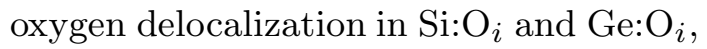
in the plane perpendicular to the $\mathrm{Si}-\mathrm{Si}$ $(\mathrm{Ge}-\mathrm{Ge})$ axis.

between two semiconductor atoms and remains quantum delocalized between them. The

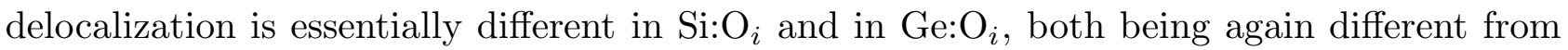
the trivial harmonic motion around a well defined geometry. In the case of silicon $[7,8]$, oxygen is delocalized in the close neighborhood of the original silicon-silicon bond center (BC) position, whereas in germanium the defect can be very precisely accounted for by an 
almost free rotor. As a consequence of the different geometries the low energy excitation spectra are quite different. In addition, in the infrared spectral region, interstitial oxygen in silicon induces a backbond-stretching resonance at $517 \mathrm{~cm}^{-1}$ which has no counterpart in Ge: $\mathrm{O}_{i}$, while in germanium there is a $\nu_{2}$ bending mode in the infrared that in $\mathrm{Si}: \mathrm{O}_{i}$ appears among the low-energy excitations.

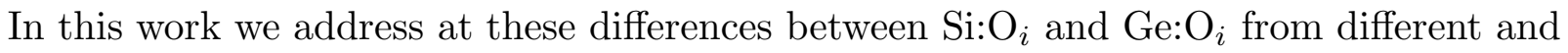
complementary points of view. With the help of total energy calculations, we determine the "equilibrium" configuration of $\mathrm{Si}: \mathrm{O}_{i}$ and $\mathrm{Ge}: \mathrm{O}_{i}$ and analyze the low energy excitations experiments. The atomic vibration and infrared spectra associated to the defect are also calculated. We find vibrational modes which have never been directly observed experimentally. We show that these modes, in combination with the high frequency stretching modes, are responsible for the experimentally observed modes induced by oxygen at $1751 \mathrm{~cm}^{-1}$ and $1260 \mathrm{~cm}^{-1}$ in

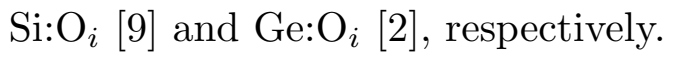

\section{Atomic configuration and far infrared analysis}

In Fig. 2 the results of the analysis of the low energy excitations of the interstitial centers are shown. In the case of $\mathrm{Si}: \mathrm{O}_{i}$ the theoretical results of Yamada-Kaneta et al. [7] along with the experimental results of the far infrared measurements of Bosomworth et al. [6] are displayed, supporting the image of Fig. 1. In the case of $\mathrm{Ge}: \mathrm{O}_{i}$, the experimental results of Gienger et al. [3] are drawn along with the results of our hindered rotor theoretical analysis, where the Hamiltonian of an elastic rotor [10] $\left(H_{\mathrm{O}}=B l^{2}-D l^{4}, l\right.$ being the angular momentum $)$ is perturbed by an angular-dependent potential $H^{\prime}=(A / 2) \cos (6 \phi)$ with an amplitude $A \approx 0.6$ $\mathrm{meV}$. The model is solved exactly to the required precision, giving a remarkable agreement with experiments, which supports the picture of Fig 1. It has to be stressed, however, that higher harmonics of the angular potential affect very little the displayed results, leaving the hindering potential (and thus the angular energy barrier, and the position and number of minima) indetermined in that respect (the measurement of the splitting of the $l= \pm 6$ levels would resolve the indetermination). This is illustrated in Fig. 2, where an added $\cos (12 \phi)$ term is considered. Note the possible qualitative differences.

Fig. 2: Low-energy excitations of $\mathrm{Si}: \mathrm{O}_{i}$ and $\mathrm{Ge}: \mathrm{O}_{i}$. For $\mathrm{Si}: \mathrm{O}_{i}$ far-infraredabsorption data [6] are compared with the fit of Yamada-Kaneta et al. [7] based on the anharmonic potential well coupled to the $\nu_{3}$ vibration mode. Also displayed is the renormalized potential for the ground

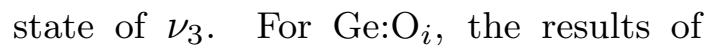
phonon spectroscopy of Gienger et al. [3] are compared with the results of the hindered rotor model. Also shown are three potentials compatible with the data, differing in the amplitude of the $\cos (12 \phi)$ term (zero, negative, or positive, respectively). 
It is important to note in Fig. 2 the different energy scales involved. The angular delocalization of oxygen in germanium gives rise to low lying rotation excitations which are essentially decoupled from the vibrational modes at a different energy scale (see below). In silicon, radial and angular excitations are non-trivially mixed in a common energy scale.

First-principles total-energy calculations have been performed in the manner described elsewhere [8], using a cluster-Hartree-Fock approximation. The results of the calculations, carefully considering relaxations up to second nearest neighbor atoms of oxygen, are shown in Fig. 3 for the radial dependence. The size of the barrier for the radial motion in Ge: $\mathrm{O}_{i}$ localizes the oxygen distance to $\mathrm{BC}$ around $r_{\mathrm{O}}=0.58 \AA$. The amplitude of the $\cos (6 \phi)$ harmonic for the angular dependence of the potential has been calculated to be of a few tenths of a meV for $\mathrm{Ge} \mathrm{O}_{i}$, in qualitative agreement with the model described above. These results give additional support to the picture of Fig. 1. In the equilibrium geometry the Ge-O-Ge (Si-O-Si) angle is $140^{\circ}\left(180^{\circ}\right)$ and the Ge-O (Si-O) distance $1.70 \AA(1.56 \AA)$.

Fig. 3: Radial potential for the oxygen motion for $\mathrm{Si}: \mathrm{O}_{i}$ and $\mathrm{Ge}: \mathrm{O}_{i}$ obtained from first principles relaxing up to second nearest neighbors of oxygen for every point. There is a flat region in the $\mathrm{Si}: \mathrm{O}_{i}$ case [8] compatible with the potential in Fig. 2.

\section{Vibrational modes and infrared absorption}

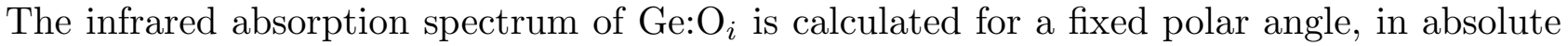
units [11] and the results are shown in Fig. 4 (the results for $\mathrm{Si}_{i} \mathrm{O}_{i}$ have been published elsewhere [8]). Four distinct features appear at 877, 416, 230, and $0 \mathrm{~cm}^{-1}$, respectively. The latter corresponds to the free rotation of oxygen and the other three correspond to the $\nu_{3}$ stretching, $\nu_{1}$ bending, and $\nu_{2}$ rocking modes of the $\mathrm{Ge}_{2} \mathrm{O}$ unit [1] (see below). The never ob-

Fig. 4: Infrared absorption coefficient

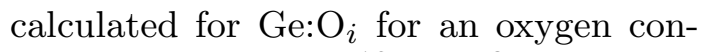
centration $[\mathrm{O}]=10^{18} \mathrm{~cm}^{-3}$. The inset shows a magnification of the spectral region corresponding to the upper Ge phonon bands, compared with the equivalent in $\mathrm{Si}: \mathrm{O}_{i}$. 
served $\nu_{2}$ peak appears as a resonance in the germanium continuum and corresponds to the radial vibration of the rotor. Indeed, the centrifugal distortion coefficient $D=4 B^{3} / \hbar^{2} \omega^{2}[10]$ in the hindered rotor model gives a value for $\omega$ of the magnitude of the frequency of this $\nu_{2}$ mode (the exact value depends on the amplitude of the $\cos (12 \phi)$ term). The position of this $\nu_{2}$ feature is not as well defined by our model as the others, being more sensitive to small changes in the dynamical matrix.

Fig. 5: Displacement-displacement correlation functions, $\left\langle u_{i} u_{j}\right\rangle(\omega)$ of oxygen and neighboring germanium atoms in $\mathrm{Ge}: \mathrm{O}_{i}$.

Although the asymmetric streching mode at $877 \mathrm{~cm}^{-1}$ has been very well characterized experimentally as a main absorption peak at $860 \mathrm{~cm}^{-1}$, the mode at $416 \mathrm{~cm}^{-1}$ (parallel to the $596 \mathrm{~cm}^{-1}$ mode in $\mathrm{Si}: \mathrm{O}_{i}$ ) has never been observed experimentally. This is due to the weakness of this feature and also to the fact that the infrared spectrum of crystalline germanium shows a two-phonon infrared absorption in this frequency range [12] that can hide the absorption of $\mathrm{O}_{i}$ at the usual concentration. Nevertheless, the combination of this mode with the asymmetric stretching at $877 \mathrm{~cm}^{-1}$ has been observed experimentally at 1260 $\mathrm{cm}^{-1}[2]$, in the same manner as in $\mathrm{Si}: \mathrm{O}_{i}$ the non-infrared active mode at $596 \mathrm{~cm}^{-1}$ combines $^{-1}$ with the asymmetric stretching one to give the $1751 \mathrm{~cm}^{-1}$ band [9]. This assignment of the combination mode is soundly supported by the agreement of the isotope shifts predicted by the theory and the corresponding experimental results. These data have already been presented for $\mathrm{Si}_{i} \mathrm{O}_{i}[8,13]$ and will be published elsewhere for ${\mathrm{Ge}: \mathrm{O}_{i}}_{[14]}$.

The displacement-displacement correlation functions $\left\langle u_{i} u_{j}\right\rangle(\omega)$ of atoms in the vicinity of the defect are shown in Fig. 5, confirming the assignments made above. The inset of

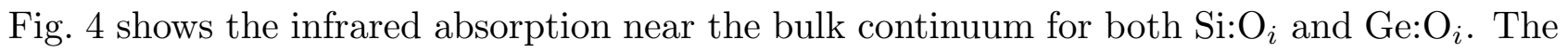
figure shows remarkable differences between these two spectra. The asymmetric resonance in $\mathrm{Si}: \mathrm{O}_{i}$ at $517 \mathrm{~cm}^{-1}$, absent in $\mathrm{Ge}: \mathrm{O}_{i}$, is due to the backbonding relaxation caused by the incorporation of oxygen. This relaxation is larger in silicon than in germanium. For a better understanding of this feature we have calculated the displacement-displacement correlation function between the silicon atoms forming the backbonds. Results of the calculations are shown in Fig.6. These results show very explicitly the origin of the modes at 517 and $596 \mathrm{~cm}^{-1}$ (416 $\mathrm{cm}^{-1}$ in Fig. 5) in silicon (germanium). The mode at $517 \mathrm{~cm}^{-1}$ is a silicon optic mode (transverse to the defect axis), with a higher frequency due to the backbonding compression. 
The mode at $596 \mathrm{~cm}^{-1}\left(416 \mathrm{~cm}^{-1}\right)$ is a (longitudinal) optic mode split-off the band due to the presence of the stronger $\mathrm{Si}-\mathrm{O}-\mathrm{Si}$ ( Ge-O-Ge) bond in the semiconductor lattice.

Fig. 6: Displacement-displacement correlation functions of silicon atoms forming backbonds in $\mathrm{Si}: \mathrm{O}_{i}$.

\section{Concluding remarks}

Total energy calculation, analysis of the low energy excitations and calculation of the infrared spectra have allowed us to fully understand the similarities and differences of interstitial oxygen in silicon and germanium. It is found that, in spite of the similarity of the defects, there are fundamental differences that manifest themselves in the low energy excitation spectra and appear as subtle but relevant details in the infrared absorption.

We thank A. J. Mayur for bringing our attention to the problem and B. Pajot for fruitful discussions. This work has been supported in part by the DGICYT through grant PB92-0169.

\section{References}

[1] See R.C.Newman, Infrared Studies of Crystal Defects, Taylor and Francis, London, 1973.

[2] B. Pajot and P. Clauws, Proc. of the $18^{\text {th }}$ International Conference on the Physics of Semiconductors, ed. O. Engström, (World Scientific, Singapore, 1987) p. 911.

[3] M. Gienger, M. Glaser and K. Laßmann, Solid State Commun. 86, 285 (1993).

[4] A. Lizón-Nordström and F. Ynduráin, Solid State Commun. 89, 819 (1994).

[5] A. J. Mayur, M. D. Sciacca, M. K. Udo, A. K. Ramdas, K. Itoh, J. Wolk, and E. E. Haller, Phys. Rev. B 49, 16293 (1994).

[6] D. R. Bosomworth, W. Hayes, A. R. L. Spray, and G. D. Watkins, Proc. Roy. Soc. Lond. A 317, 133 (1970).

[7] H. Yamada-Kaneta, C. Kaneta, and T. Ogawa, Phys. Rev. B 42, 9650 (1990).

[8] E. Artacho, A. Lizón-Nordström and F. Ynduráin, Phys. Rev. B 51, 7862 (1995); E. Artacho and F. Ynduráin, Proc. of the 22 ${ }^{\text {nd }}$ International Conference on the Physics of Semiconductors, ed. D. J. Lockwood, (World Scientific, Singapore, 1995) p. 2459.

[9] B. Pajot, H.J. Stein, B. Cales and C. Naud, J. Electrochem. Soc. 132, 3034 (1985).

[10] G. Herzberg, Infrared and Raman Spectra of Diatomic Molecules, (D. Van Nostrand Company, Inc., 1945) p. 104.

[11] Model dynamical-matrix calculation for a single $\mathrm{O}$ impurity in an infinite semiconductor, described in Ref. 8. The absolute units are obtained as in E. Martínez and M. Cardona, Phys. Rev. B 28, 880 (1983).

[12] R. Tubino, L. Piseri, and G. Zerbi, J. Chem. Phys. 56, 1022 (1972); K. Winer and M. Cardona, Phys. Rev. B 35, 8189 (1987) and references therein.

[13] B. Pajot and P. Cales, Mat. Res. Symp. Proc. 59, 39 (1986). B. Pajot, E. Artacho, C. A. J. Ammerlaan, and J. M. Spaeth, to appear in J. Phys.: Condens. Matter.

[14] E. Artacho, F. Ynduráin, B. Pajot, L. I. Khirunenko, R. Ramírez, and C. P. Herrero, to be published. 


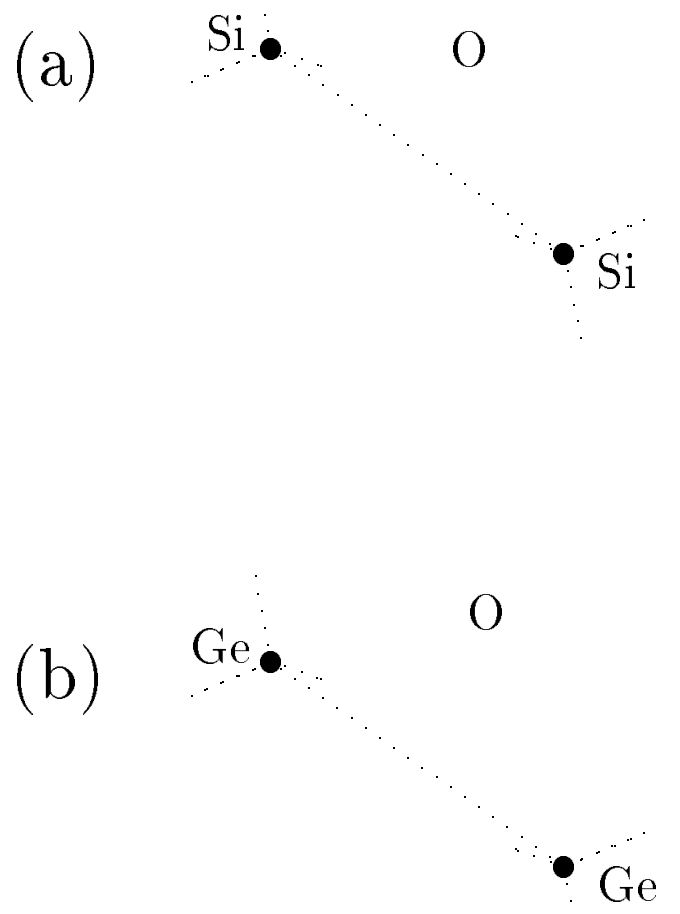



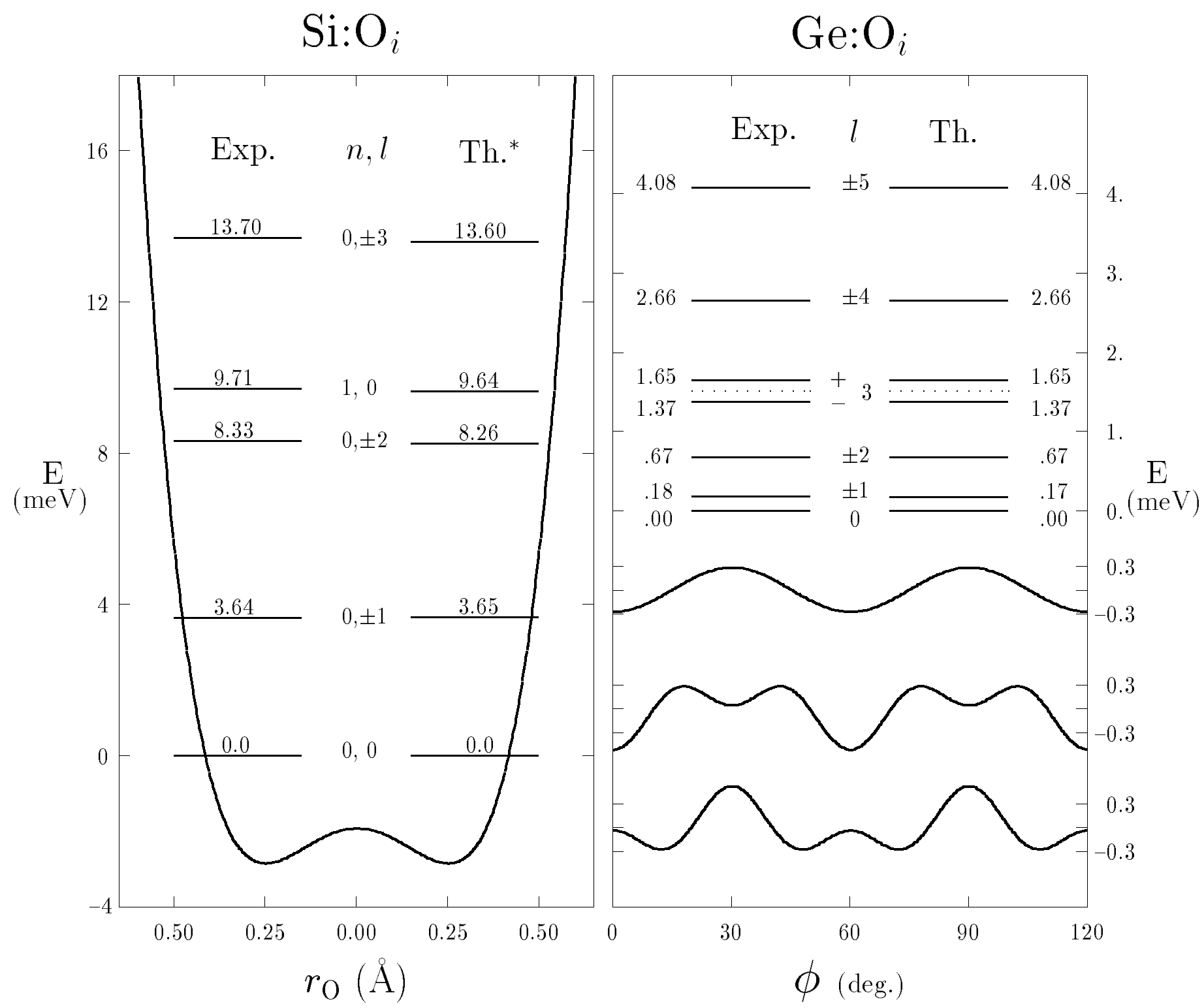


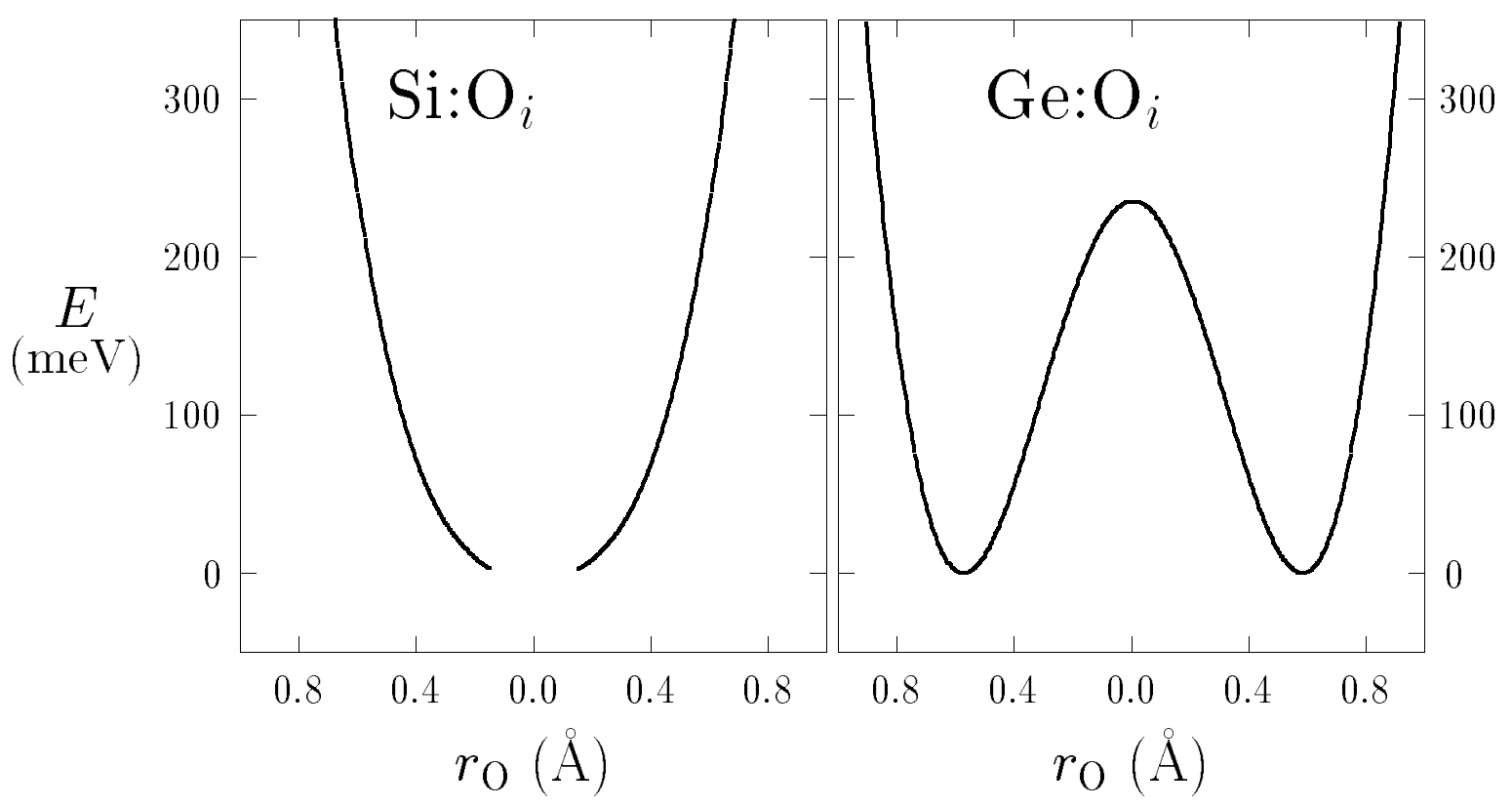




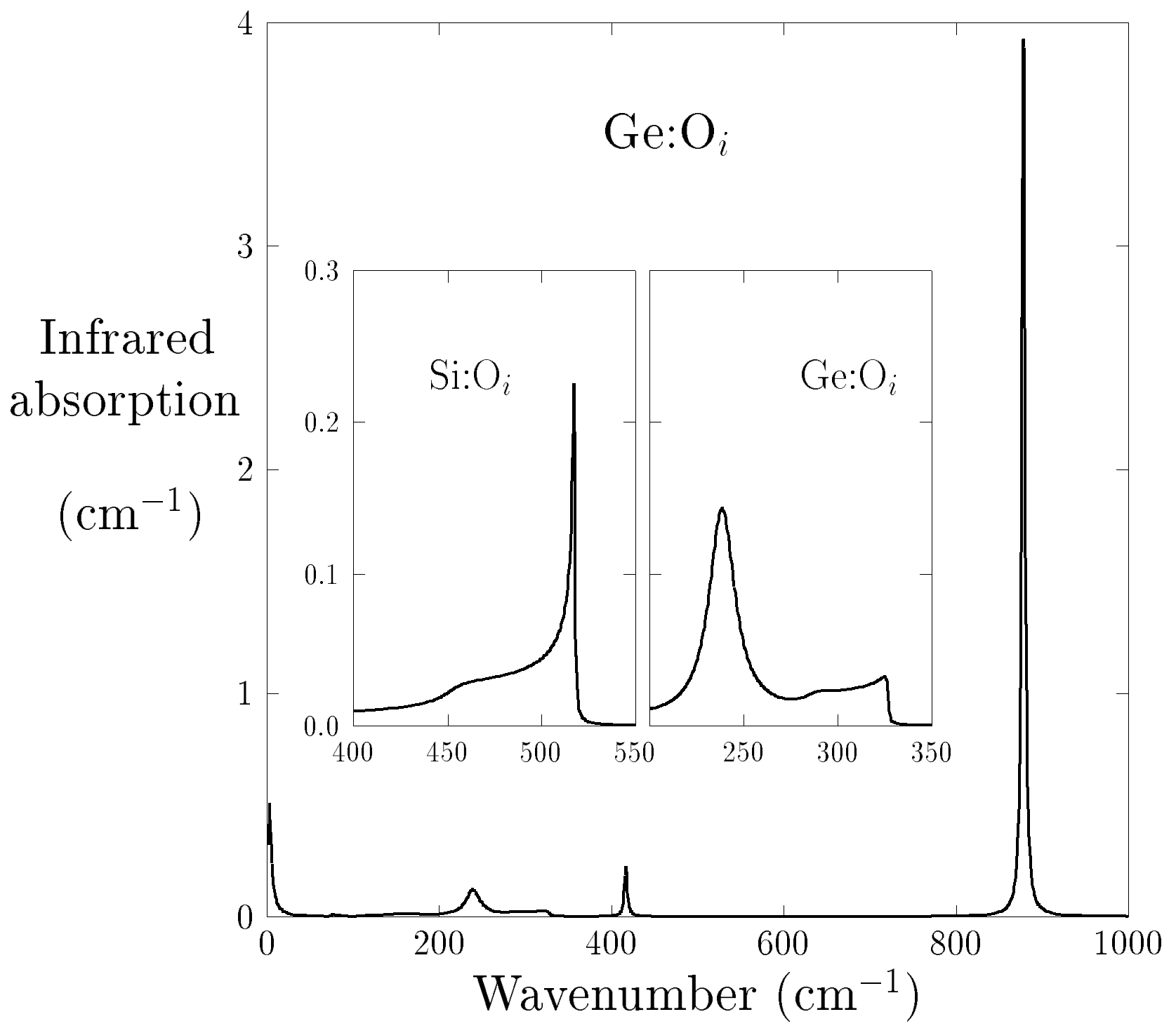




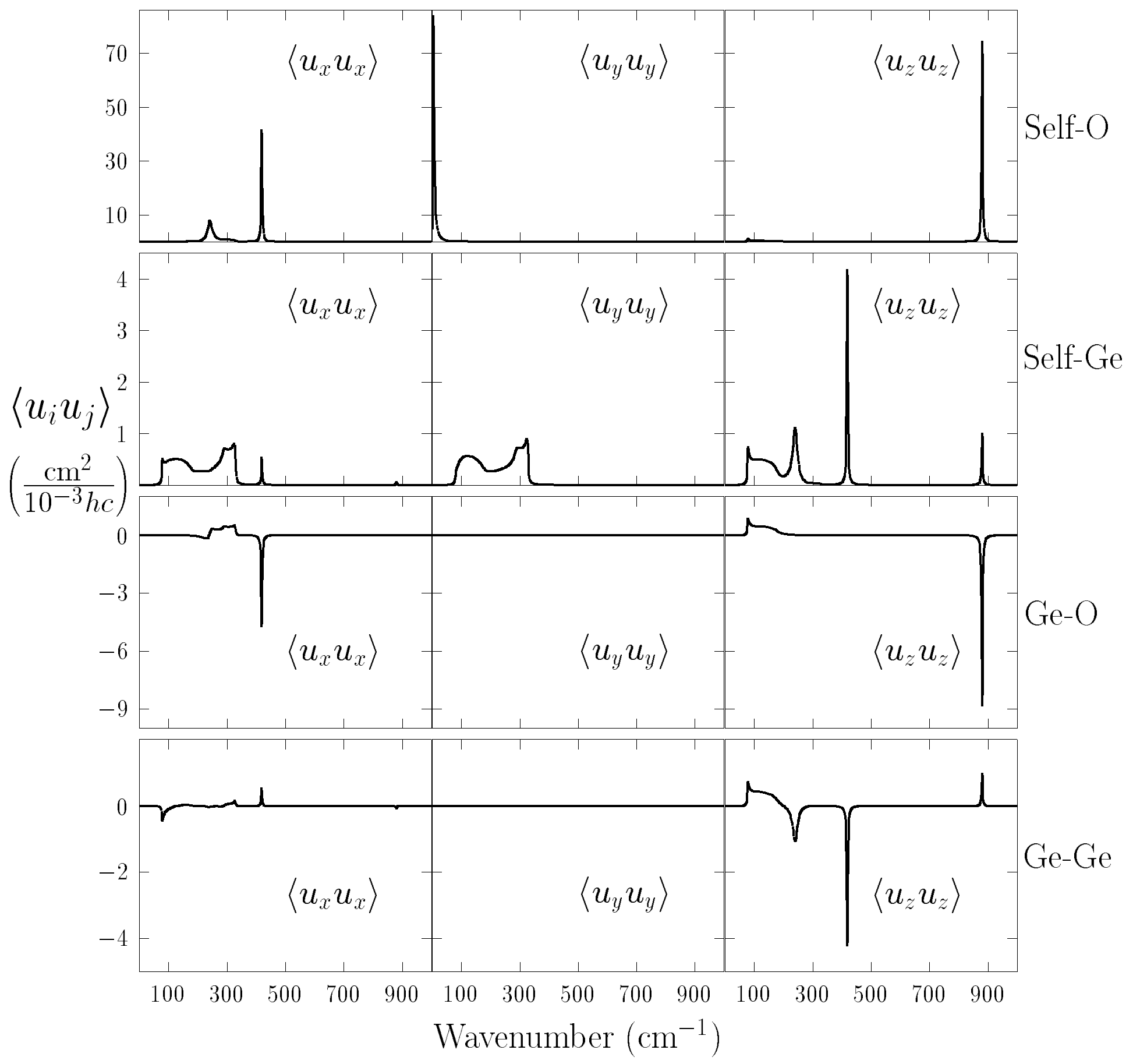




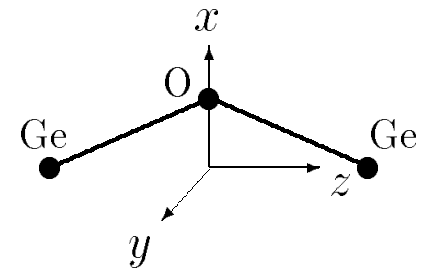




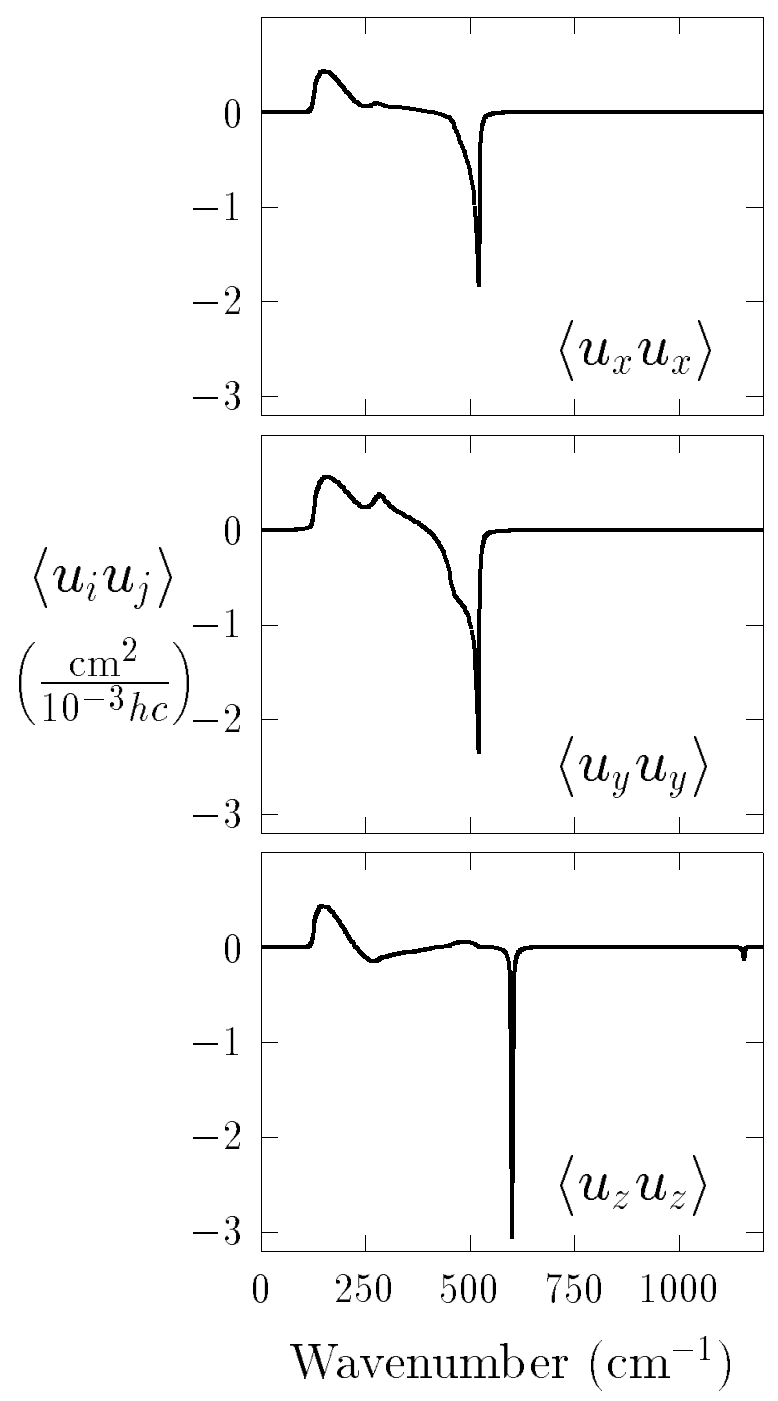

